\title{
Acute left ventricular dysfunction following gemtuzumab ozogamicin in two children with AML
}

\author{
Kevin McNerney ${ }^{1}$, Katelyn Oranges ${ }^{1}$, Alix Seif ${ }^{2}$, Benjamin Oshrine ${ }^{3}$, Kelly Getz ${ }^{1}$, and \\ Richard Aplenc ${ }^{4}$ \\ ${ }^{1}$ The Children's Hospital of Philadelphia \\ ${ }^{2}$ Children's Hospital of Philadelphia \\ ${ }^{3}$ Johns Hopkins All Children's Hospital \\ ${ }^{4}$ The Children's Hospital of Philadelphia
}

August 23, 2020

\begin{abstract}
Gemtuzumab ozogamicin (GO) is an anti-CD33 antibody-tumor antibiotic conjugate with proven efficacy in pediatric and adult patients with CD33+ acute myeloid leukemia (AML). Adverse effects commonly associated with GO include hyperbilirubinemia, elevated transaminases, and sinusoidal obstruction syndrome (SOS). Cardiotoxicity has not been a commonly described adverse event. We describe two pediatric patients with relapsed/refractory AML who received fractionated GO monotherapy and subsequently developed severe acute left ventricular dysfunction. Both patients achieved remission, recovered cardiac function with medical therapy, and tolerated subsequent stem cell transplantation.
\end{abstract}

Acute left ventricular dysfunction following gemtuzumab ozogamicin in two children with AML

Kevin McNerney ${ }^{1}$; Katelyn Oranges ${ }^{1}$, Alix Seif ${ }^{1,2}$, Benjamin Oshrine ${ }^{3}$, Kelly Getz ${ }^{2}$, Richard Aplenc ${ }^{1,2 *}$

${ }^{1}$ Division of Oncology, Children's Hospital of Philadelphia, Philadelphia, PA, 19104

${ }^{2}$ Perelman School of Medicine, University of Pennsylvania, Philadelphia, PA, 19104

${ }^{3}$ Johns Hopkins All Children's Hospital, St. Petersburg, FL, 33701

${ }^{*}$ Correspondence to:

Richard Aplenc, MD, PhD, Division of Oncology, Children's Hospital of Philadelphia, 3501 Civic Center Blvd, Room 4018, Philadelphia, PA 19104. Tel: 267-426-7252, Email: aplenc@email.chop.edu

Text Word Count: 1200

Abstract Word Count: 84

Brief Running Title: Gemtuzumab and cardiotoxicity in pediatric AML

Tables: 1

Figures: 1

\begin{tabular}{ll}
\hline Abbreviations & Abbreviations \\
\hline AUS & Abdominal ultrasound \\
AML & Acute myeloid leukemia
\end{tabular}




\begin{tabular}{ll}
\hline Abbreviations & Abbreviations \\
\hline BNP & Brain natriuretic peptide \\
BMBA & Bone marrow aspirate and biopsy \\
BMT & Bone marrow transplant \\
ECHO & Echocardiogram \\
GO & Gemtuzumab Ozogamicin \\
ICU & Intensive Care Unit \\
LVEF & Left ventricular ejection fraction \\
LVSF & Left ventricular shortening fraction \\
LVSD & Left ventricular systolic dysfunction \\
MR & Mitral regurgitation \\
PSCT & Peripheral stem cell transplant \\
r/r & Relapsed/refractory \\
SOS & Sinusoidal obstruction syndrome \\
\hline
\end{tabular}

Keywords: acute myeloid leukemia; immunotherapy; cardiotoxicity

\section{ABSTRACT}

Gemtuzumab ozogamicin (GO) is an anti-CD33 antibody-tumor antibiotic conjugate with proven efficacy in pediatric and adult patients with CD33+ acute myeloid leukemia (AML). Adverse effects commonly associated with GO include hyperbilirubinemia, elevated transaminases, and sinusoidal obstruction syndrome (SOS). Cardiotoxicity has not been a commonly described adverse event. We describe two pediatric patients with relapsed/refractory AML who received fractionated GO monotherapy and subsequently developed severe acute left ventricular dysfunction. Both patients achieved remission, recovered cardiac function with medical therapy, and tolerated subsequent stem cell transplantation.

\section{INTRODUCTION}

Gemtuzumab ozogamicin (GO) is an anti-CD33 antibody-tumor antibiotic conjugate used in the treatment of both de novo and relapsed/refractory (r/r) pediatric acute myeloid leukemia (AML)with demonstrated improvements in event free survival and relapse risk $^{1}$. In $\mathrm{r} / \mathrm{r}$ pediatric AML, GO has been used both as monotherapy (2.5-10 mg $/ \mathrm{m}^{2}{ }^{2-45}$ or as $9 \mathrm{mg} / \mathrm{m}^{2}$ fractionated into three $3 \mathrm{mg} / \mathrm{m}^{2}$ doses $\left.{ }^{6,7}\right)$ and in combination with chemotherapy ${ }^{5-8}$.

The main toxicities associated with GO include myelosuppression, hyperbilirubinemia, transaminitis, and sinusoidal obstruction syndrome (SOS). The incidence of SOS is directly associated with the absolute single dose of GO, and fractionation of GO doses has been shown to reduce SOS incidence ${ }^{6,7,9,10}$.

Cardiotoxicity related to GO is rare, and only reported in pre-treated r/r AML patients receiving unfractionated dosing ${ }^{2,5}$. One study using two $7.5 \mathrm{mg} / \mathrm{m}^{2}$ GO doses in 30 patients with $\mathrm{r} / \mathrm{r}$ pediatric AML found 4/30 patients had adverse effects in left ventricle shortening fraction (LVSF) with 2/4 having grade 3 or 4 toxicity $^{2}$. Another study using unfractionated GO in $\mathrm{r} / \mathrm{r}$ pediatric AML observed one death from worsening of pre-existing cardiomyopathy with $\mathrm{SOS}^{5}$.

We have used unfractionated GO monotherapy in two pediatric patients with r/r AML that subsequently developed life-threatening left ventricular systolic dysfunction (LVSD). Herein, we present their vignettes along with echocardiography and laboratory findings to phenotype this rarely-described toxicity of an increasingly commonly used immunotherapy.

\section{CASE DESCRIPTIONS}

\section{Patient 1}


A 23-month-old female with CD33+ AML was initially treated with the AML-BFM 2012 intermediate risk group protocol. Prior to receiving AML therapy, she was noted to have a mildly dilated LV with mitral regurgitation (MR) for which enalapril and furosemide were used during induction. Six months into maintenance therapy she developed an isolated medullary relapse and was re-induced (Table 1). A bone marrow biopsy and aspirate (BMBA) on day 15 of re-induction chemotherapy showed aplastic marrow with $88 \%$ blasts, prompting a decision to start GO for presumed chemorefractory disease.

Her pre-GO cumulative anthracycline exposure was $400 \mathrm{mg} / \mathrm{m}^{2}$ and an echocardiogram (ECHO) showed lownormal left ventricular ejection fraction (LVEF) of $52 \%$ and low-normal LVSF of $26 \%$ with no MR (Figure 1). She received $3 \mathrm{mg} / \mathrm{m}^{2} \mathrm{GO}$ on days 1,4 , and 7 , and then underwent repeat BMBA that was hypoplastic and minimal residual disease (MRD)-negative. She then received an additional three GO doses $\left(3 \mathrm{mg} / \mathrm{m}^{2} /\right.$ dose $)$ on days 15, 18, and 21 and subsequently developed prolonged pancytopenia but exhibited no symptoms of heart failure. At day 62 after GO, she initiated azacytidine and venetoclax as bridging therapy to planned bone marrow transplant (BMT).

At day 82 after GO, a routine ECHO revealed a decrease of LVEF to $40 \%$ and LVSF to $16 \%$, a moderately dilated LV $(4.41 \mathrm{~cm}$, from $3.5 \mathrm{~cm}$ pre-GO), mild MR, and a brain natriuretic peptide (BNP) of 1592 $\mathrm{pg} / \mathrm{mL}$. She was started on furosemide and enalapril, followed by carvedilol. Two weeks later, she developed pallor and fatigue requiring admission. An ECHO showed a severely dilated LV, LVEF of $32 \%$, and LVSF of $16 \%$. Her carvedilol dose was decreased, and digoxin was started. She completed 4 cycles of azacytidine and venetoclax during which time her LVEF improved to $54 \%$ and LVSF improved to $30 \%$ with mild LV dilation.

Seven months post-GO, she remained in an MRD-negative remission and underwent unrelated donor BMT, after which she had stable cardiac function and did not develop SOS. Her AML is in remission 9 months post-BMT and she is maintained on a regimen of carvedilol, digoxin, spironolactone, and enalapril with stable cardiac function.

\section{Patient 2}

A 3-year-old male with CD33+ AML received upfront therapy on AAML1031 Arm B (bortezomib arm), and relapsed with a scalp chloroma seven months post-diagnosis. He was re-induced (Table 1), but following re-induction a BMBA demonstrated $35 \%$ blasts. He then received clofarabine, topotecan, thiotepa, and vinorelbine, and his MRD decreased to $1 \%$. He underwent unrelated donor peripheral stem cell transplant (PSCT) followed by azacytidine for nine cycles before developing a maxillary sinus chloroma and $33 \%$ marrow involvement with CD33+ myeloid blasts. This second relapse prompted the decision to pursue GO.

A pre-GO ECHO showed normal LVEF of $60 \%$ and LVSF of $31 \%$ (Figure 1$)$. He received GO $\left(3 \mathrm{mg} / \mathrm{m}^{2} /\right.$ dose) on days 1, 4, and 8 with concurrent radiotherapy for the chloroma. BMBA on day 11 showed hypocellularity without leukemic blasts. He received two additional doses of GO on days 15 and 18 for $15 \mathrm{mg} / \mathrm{m}^{2}$ total. He then developed respiratory distress, hypotension, altered mental status, and a distended abdomen requiring transfer to the intensive care unit (ICU) while afebrile with negative blood cultures. An ECHO showed a severely diminished LVEF of $21 \%$, LVSF of $12 \%$, and mild LV dilation. An abdominal ultrasound (AUS) showed hepatomegaly but normal portal venous flow. Labwork showed a BNP of $6373 \mathrm{pg} / \mathrm{mL}$ and a bilirubin that peaked at $2.9 \mathrm{mg} / \mathrm{dL}$. He was started on dobutamine, milrinone, and epinephrine for cardiogenic shock. His epinephrine and dobutamine were stopped after 2 days, milrinone was stopped after 14 days, and he initiated enalapril and spironolactone. His LVEF improved to 55\%, and LVSF to $29 \%$ within 12 days of the ICU transfer. A BMBA two months post-GO remained MRD negative, and an ECHO three months post-GO showed stable LV function. As a bridge to a second SCT, he received a cycle of decitabine.

4 months following GO, he underwent BMT. On day +16 he developed hepatomegaly and a new cardiac gallop; ECHO showed a decrease in LVEF to $45 \%$ and LVSF to $25 \%$, his BNP was $8167 \mathrm{pg} / \mathrm{ml}$, and he was transferred to the ICU where furosemide and milrinone were started. AUS showed hepatomegaly with normal flow in the portal vein. Five days later, milrinone was stopped and within 1 week his BNP trended down to 396 and his LVEF and LVSF normalized. 50 days post-BMT an ECHO and BNP were repeated 
and remained normal. Unfortunately, 21 months post-BMT, he had a bone marrow/extramedullary relapse and died 3 months later.

DISCUSSION We present vignettes of two pediatric patients with $\mathrm{r} / \mathrm{r}$ AML who developed acute LV dysfunction following $15-18 \mathrm{mg} / \mathrm{m}^{2}$ of fractioned GO monotherapy, suggesting a potential relationship between GO and cardiotoxicity in pre-treated AML patients. Both patients had significant prior anthracycline exposure but low-normal to normal cardiac function before GO administration. Their cardiac dysfunction was characterized by severely decreased systolic and diastolic function, elevated BNPs, and improvement with medical therapy.

Patient 2 was critically ill without alternative clinical explanation when LVSD was identified while Patient 1 was initially asymptomatic, suggesting the utility of routine ECHO monitoring in patients with AML receiving GO-monotherapy at these doses. Both patients achieved remission following three $3 \mathrm{mg} / \mathrm{m}^{2}$ doses of GO, tolerated full-intensity SCT, did not develop SOS, and maintained post-SCT remissions for 9 months and counting for Patient 1, and 21 months for Patient 2. Given that fractionation was hypothesized to reduce toxicity from GO, it is important to note that the cardiac toxicity encountered at doses of $15-18 \mathrm{mg} / \mathrm{m}^{2}$ was not prevented by fractionation, although SOS did not occur and toxicity did not preclude SCT. While cardiotoxicity is not commonly described with GO, this and prior reports of worsening LVSF following $\mathrm{GO}^{2,5}$ suggest the need for cardiac surveillance following GO administration. Future studies evaluating cardiotoxicity following GO may deepen our understanding of this clinically important toxicity and guide optimal monitoring strategies for these patients.

\section{ACNKOWLEDGEMENTS}

We would like to thank all that contributed to and supported this project. KM reviewed the medical histories, wrote and revised the manuscript, created the table and figure. KG compiled echocardiogram data. KG, $\mathrm{KO}$, AS, and BO provided critical revisions to the manuscript. RA conceptualized the project, provided guidance on the development of the manuscript, and provided revisions. No authors have conflicts of interest to disclose.

\section{CITATIONS}

1. Gamis AS, Alonzo TA, Meshinchi S, et al. Gemtuzumab Ozogamicin in Children and Adolescents With De Novo Acute Myeloid Leukemia Improves Event-Free Survival by Reducing Relapse Risk: Results From the Randomized Phase III Children's Oncology Group Trial AAML0531. J Clin Oncol . 2014;32(27):3021-3032. doi:10.1200/JCO.2014.55.3628

2. Zwaan CM, Reinhardt D, Zimmerman M, et al. Salvage treatment for children with refractory first or second relapse of acute myeloid leukaemia with gemtuzumab ozogamicin: results of a phase II study. $B r J$ Haematol . 2010;148(5):768-776. doi:10.1111/j.1365-2141.2009.08011.x

3. Arceci RJ. Safety and efficacy of gemtuzumab ozogamicin in pediatric patients with advanced CD33+ acute myeloid leukemia. Blood . 2005;106(4):1183-1188. doi:10.1182/blood-2004-10-3821

4. Brethon B, Auvrignon A, Galambrun C, et al. Efficacy and tolerability of gemtuzumab ozogamicin (anti-CD33 monoclonal antibody, CMA-676, Mylotarg@) in children with relapsed/refractory myeloid leukemia.BMC Cancer . 2006;6(1):172. doi:10.1186/1471-2407-6-172

5. Niktoreh N, Lerius B, Zimmermann M, et al. Gemtuzumab ozogamicin in children with relapsed or refractory acute myeloid leukemia: a report by Berlin-Frankfurt-Münster study group. Haematologica . 2019;104(1):120-127. doi:10.3324/haematol.2018.191841

6. Brethon B, Yakouben K, Oudot C, et al. Efficacy of fractionated gemtuzumab ozogamicin combined with cytarabine in advanced childhood myeloid leukaemia. Br J Haematol . Published online August 2008. doi:10.1111/j.1365-2141.2008.07370.x 
7. Liu AP-Y, Leung AW-K, Cheuk DK-L, Lee V, Ha S-Y. Gemtuzumab Ozogamicin Containing Chemotherapy for Relapsed or Refractory Acute Myeloid Leukemia (AML) in Children: J Pediatr Hematol Oncol . 2018;40(2):163-168. doi:10.1097/MPH.0000000000001010

8. Aplenc R, Alonzo TA, Gerbing RB, et al. Safety and Efficacy of Gemtuzumab Ozogamicin in Combination With Chemotherapy for Pediatric Acute Myeloid Leukemia: A Report From The Children's Oncology Group.J Clin Oncol . 2008;26(14):2390-2395. doi:10.1200/JCO.2007.13.0096

9. Larson RA, Sievers EL, Stadtmauer EA, et al. Final report of the efficacy and safety of gemtuzumab ozogamicin (Mylotarg) in patients with CD33-positive acute myeloid leukemia in first recurrence. Cancer . 2005;104(7):1442-1452. doi:10.1002/cncr.21326

10. Taksin A-L, Legrand O, Raffoux E, et al. High efficacy and safety profile of fractionated doses of Mylotarg as induction therapy in patients with relapsed acute myeloblastic leukemia: a prospective study of the alfa group. Leukemia . 2007;21(1):66-71. doi:10.1038/sj.leu.2404434

TABLE 1 Legend:

TABLE 1: Characteristics of patient 1 and 2. ADxE, cytarabine, liposomal daunorubicin, etoposide; haM, high-dose cytarabine/mitoxantrone; AI/2 CDA, cytarabine/ idarubicin/2-chloro-2-deoxyadenosine; HAE, high-dose cytarabine/etoposide; 6-TG, 6-thioguanine; AraC, cytarabine; FLAG-Ida, fludarabine, high-dose cytarabine, G-CSF, and idarubicin; ATG, anti-thymocyte globulin; ADE, cytarabine, daunorubicin, etoposide; AE, cytarabine, etoposide; AM, cytarabine, mitoxantrone. ${ }^{*} \mathrm{MRD}$ not evaluable due to hypocellular marrow.

\section{FIGURE 1 LEGEND:}

FIGURE 1: Cardiac Function vs Time Post-GO for Patients 1 and 2A. Left ventricle ejection fraction (LVEF) as measured by M-Mode for Patient 1 (blue circles) and Patient 2 (red squares) vs time in days from gemtuzumab ozogamicin (GO) with clinical highlights.B. LV systolic function (LVSF) measurements over time measured with M-Mode. C. LV diastolic volume in milliliters $(\mathrm{mL})$ over time. D. LV Mass z-score over time. E. LV Mass Index expressed as grams (g)/height (ht $)^{2.7}$. F\&G.LV transmitral early peak velocity (E) to early diastolic mitral annulus velocity $\left(e^{\prime}\right)$ ratios. $\mathrm{E} / e^{\prime}$ ratios $>14$ are one feature of diastolic dysfunction. H. B-Natriuretic Peptide measurements over time in picograms $(\mathrm{pg}) / \mathrm{ml}$

\section{Hosted file}

Table 1_Final.docx available at https://authorea.com/users/343385/articles/476652-acute-leftventricular-dysfunction-following-gemtuzumab-ozogamicin-in-two-children-with-aml 

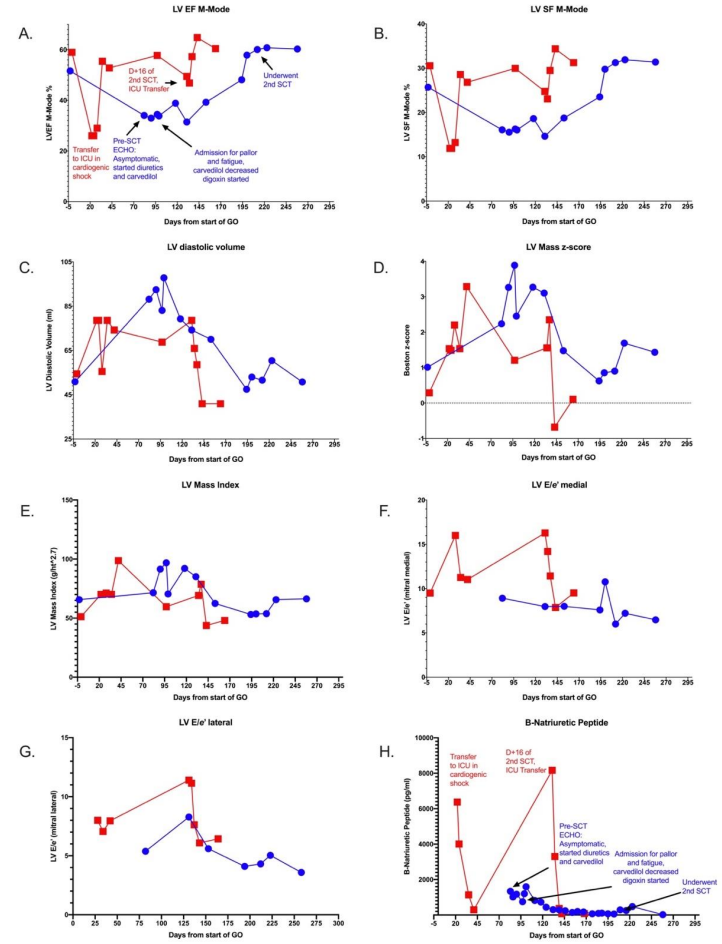

FIGURE 1: Cardiac Function vs Time Post-GO for Patients 1 and 2 A. Left ventricle ejection fraction (LVEF) as measured by M-Mode for Patient 1 (blue circles) and Patient 2 (red squares) vs time in days from gemtuzumab ozogamicin (GO) with clinical highlights. B. LV systolic function (LVSF) measurements over time measured with M-Mode. C. LV diastolic volume in milliliters $(\mathrm{mL})$ over time. D. LV Mass z-score over time. E. LV Mass Index expressed as grams (g)/height (ht) ${ }^{2.7}$. F\&G. LV transmitral early peak velocity (E) to early diastolic mitral annulus velocity $\left(e^{\prime}\right)$ ratios. E/e' ratios $>14$ are one feature of diastolic dysfunction. H. B-Natriuretic Peptide measurements over time in picograms $(\mathrm{pg}) / \mathrm{ml}$ 